\title{
Detection and recognition thresholds for five basic tastes in patients with mild cognitive impairment and Alzheimer's disease dementia
}

Minoru Kouzuki ${ }^{1 *}$, Junya Ichikawa ${ }^{1}$, Daiki Shirasagi ${ }^{1}$, Fumiya Katsube ${ }^{1}$, Yuuki Kobashi ${ }^{1}$, Hideki Matsumoto ${ }^{2}$, Huichia Chao ${ }^{2}$, Shintaro Yoshida ${ }^{2}$ and Katsuya Urakami ${ }^{1}$

\begin{abstract}
Background: Patients with Alzheimer's disease dementia (ADD) are thought to exhibit taste disorders; however, this has not been extensively studied. We investigated gustatory functions and factors affecting taste in patients with $\mathrm{ADD}$ or mild cognitive impairment (MCl) and in non-demented controls (NDCs) and evaluated associations between cognitive impairment and gustatory functions.

Methods: We recruited 29 patients with ADD, 43 with MCl, and 14 with NDCs. We obtained medical and medication history, measured salivary secretion volumes, and performed cognitive function tests, blood tests, whole-mouth gustatory tests, and dietary and gustatory questionnaires.

Results: Patients with ADD showed significantly higher recognition threshold values than NDCs $(p<0.05)$. Many individuals did not recognize umami at the maximum concentration, and this happened more frequently in patients with $\mathrm{ADD}$ or $\mathrm{MCl}$ than in NDCs. Evaluation items other than cognitive function tests did not show significant differences among the groups, but many individuals had decreased salivation, low serum zinc levels, and were on multiple medications. We found a significant correlation between recognition threshold and age $(r=0.229$, $p<0.05)$ and cognitive function test score $(r=0.268, p<0.05)$.

Conclusions: Patients with ADD showed impairment of gustatory function. Gustatory impairment in patients with $\mathrm{MCl}$ could not be confirmed. However, many individuals with $\mathrm{MCl}$ did not recognize umami, either. Our results suggest that taste disorders in elderly people with cognitive decline occur independently of factors affecting taste such as salivation, zinc levels, or prescription drugs.
\end{abstract}

Trial registration: The study was registered in the UMIN Clinical Trials Registry on February 10, 2017, with reference number UMIN000026087.

Keywords: Alzheimer disease, Mild cognitive impairment, Gustatory function, Gustatory test

\footnotetext{
* Correspondence: kouzuki@tottori-u.ac.jp

'Department of Biological Regulation, School of Health Science, Faculty of

Medicine, Tottori University, 86 Nishi-cho, Yonago 683-8503, Japan

Full list of author information is available at the end of the article
}

(c) The Author(s). 2020 Open Access This article is licensed under a Creative Commons Attribution 4.0 International License, which permits use, sharing, adaptation, distribution and reproduction in any medium or format, as long as you give appropriate credit to the original author(s) and the source, provide a link to the Creative Commons licence, and indicate if changes were made. The images or other third party material in this article are included in the article's Creative Commons licence, unless indicated otherwise in a credit line to the material. If material is not included in the article's Creative Commons licence and your intended use is not permitted by statutory regulation or exceeds the permitted use, you will need to obtain permission directly from the copyright holder. To view a copy of this licence, visit http://creativecommons.org/licenses/by/4.0/ The Creative Commons Public Domain Dedication waiver (http://creativecommons.org/publicdomain/zero/1.0/) applies to the data made available in this article, unless otherwise stated in a credit line to the data. 


\section{Background}

Olfactory function declines in patients with Alzheimer's disease dementia (ADD) [1-4], and hearing loss is a risk factor for dementia onset [5-7], indicating that there are associations between sensory disorders and dementia. In terms of the sense of taste, gustatory function has been shown to be decreased in patients with ADD [8-10], and $81.4 \%$ of patients with ADD show some eating disturbance, such as changes in appetite and eating habits [11]. Gustatory tests include the filter-paper disc method [12], the taste-strip test [13, 14], electrogustometry [12, 15], and the whole-mouth gustatory test $[16,17]$. The filterpaper disc method, the taste-strip test, and the wholemouth gustatory test are used to evaluate the identity of taste (taste quality), whereas electrogustometry can evaluate taste thresholds. A study based on the filter-paper disc method and electrogustometry has suggested that patients with ADD may exhibit taste disorder concomitantly with brain atrophy and neurodegeneration [8]. Also, another study using taste strips reported reduced taste function in patients with mild cognitive impairment $(\mathrm{MCI})$, which is a pre-dementia state [9]. However, other reports using filter-paper discs have found no deterioration of the taste function in patients with ADD [18], and there was no detection threshold disorders for sweet and sour tastes in whole-mouth gustatory tests [19]. Some diverging results may be explained by the use of different evaluation methods. For example, when patients with ADD were evaluated by filter-paper discs and then by whole-mouth gustatory tests, the whole-mouth gustatory test indicated taste deterioration, but the filter-paper discs did not [20]. In addition, comparing studies is difficult due to different taste solution concentrations and results varying between evaluations regarding detection or recognition thresholds. Moreover, many studies have evaluated only four basic tastes (sweet, salty, sour, and bitter) and have not evaluated the fifth basic taste, umami. Inability to sense umami is thought to lead to loss of appetite [21], and umami evaluation is important to assess appetite in people with dementia. In addition, other factors affecting taste function like prescription drugs, saliva secretion, and serum zinc levels need to be considered in the interpretation of taste function tests [22-26]. Also, few studies have examined taste in patients with $\mathrm{MCI}$, and, thus, it is unclear whether taste function is impaired in these patients.

The aim of this study was to conduct a detailed investigation into the detection and recognition of the five basic tastes in the taste function of patients with ADD and $\mathrm{MCI}$, which has not been sufficiently investigated in previous studies. In this research, we adopted wholemouth gustatory tests that evaluate the taste function in the whole oral cavity, and tested patients with ADD or $\mathrm{MCI}$, and non-demented controls (NDCs) using diluted solutions of the five basic tastes. In addition, we investigated several factors related to gustatory function to consider the association of gustatory function with cognitive decline.

\section{Methods \\ Patients}

Between May 2017 and December 2018, we recruited patients with ADD $(n=29)$ or MCI $(n=43)$, and NDCs $(n=14)$, from the outpatient clinic of Shinsei Hospital (Kurayoshi, Japan). All patients underwent general physical and neurological examinations, neuropsychological assessments, such as the Geriatric Depression Scale 15-item version, the Mini-Mental State Examination (MMSE), and/or the Alzheimer's Disease Assessment Scale-Cognitive Subscale (ADAS-Cog), laboratory tests, and brain imaging studies, such as brain computed tomography $(\mathrm{CT})$, magnetic resonance imaging, and single photon emission $\mathrm{CT}$, to exclude other potential causes of dementia. We included patients with ADD that met the diagnostic criteria of the Diagnostic and Statistical Manual of Mental Disorders, fifth edition [27] and patients with MCI that met the following diagnostic criteria [28]: (i) memory complaint, preferably corroborated by an informant, (ii) objective memory impairment for age, (iii) relatively preserved general cognition for age, (iv) essentially intact activities of daily living, and (v) not demented, or NDCs without evidence of cognitive impairment or psychiatric disorders at the time of clinical and neuropsychological assessments. We excluded patients who had already been diagnosed as having taste disorders.

The ethics committee of Tottori University (Yonago, Japan) and Ajinomoto (Tokyo, Japan) approved the design of this study (approval number: 1701B073), which was conducted by following the tenets of the Declaration of Helsinki. We registered the study in the UMIN Clinical Trials Registry (UMIN000026087). We explained the research protocol to the patients and guardians, who provided written informed consent before participation.

\section{Procedures}

We performed gustatory and cognitive function tests on the subjects, measured salivary flow, height, weight, blood pressure, performed blood tests, and asked participants to answer dietary and gustatory questionnaires within 1 month after obtaining the participants' consents. If blood samples were collected during routine medical examination 1 month before or after the gustatory test, blood tests were performed using that sample. We determined the number of prescribed medications taken by the participants and their medical history (hypertension, dyslipidemia, and diabetes mellitus) from medical record data. 


\section{Gustatory test}

We used the whole-mouth method by 13 stepwise two-fold dilution series of five basic tastes prepared according to previous studies: sweet $(40-0.0098 \%$ sucrose), salty (20-0.00488\% sodium chloride), umami (2.0-0.00049\% monosodium L-glutamate [MSG]), sour (1.6-0.00039\% tartaric acid), and bitter $(0.1-0.00002 \%$ quinine hydrochloride) [16, 29]. Gustatory tests were performed according to the procedure in a previous study [16]. The patients first rinsed their mouths with water, and then we applied $1 \mathrm{~mL}$ of a taste solution at the lowest concentration onto their tongue, and asked the patients to spread it around in their entire mouth before spitting it out. For each solution tested, the participants chose one of the following seven possible answers: sweet, salty, umami, sour, bitter, unidentifiable taste, or no taste. If the patient could not correctly identify the taste, in the next trial, we used the solution in the same taste category with the next higher concentration. If the patient answered correctly, we tested a different taste solution. Before testing another solution, the patients rinsed their mouths thoroughly until the previous taste disappeared. We applied the taste solutions in the following order; sweet, salty, umami, sour, and bitter. We did this to ensure that the bitter taste, which tends to remain in the oral cavity for a long time, was applied last. With increase in the concentration of the taste solution, the detection and recognition thresholds were recorded. We defined the detection threshold as the concentration at which the taste could be detected, and the recognition threshold as the concentration at which the taste could be recognized. To determine the detection threshold, we adopted the lowest concentration at which the participant answered other than "no taste." Even if participants answered that they tasted something, if they answered "no taste" when the concentration was increased after that, the previous answers were cancelled. Patients who detected the lowest concentration received 1 point. Patients who detected the highest concentration received 13 points. Patients failing to detect the highest concentration received 14 points. For the recognition threshold, we adopted the lower concentration at which the participant answered correctly twice in a row. The scoring method for the recognition threshold was set from 1 to 14 as well as with the detection threshold.

\section{Cognitive function test}

To assess cognitive function, we used the Touch Panel-type Dementia Assessment Scale (TDAS) (Nihon Kohden Corporation, Tokyo, Japan) [30]. TDAS is a modified version of the ADAS-Cog [31] in which subjects enter their answers directly into a touch-panel computer following instructions. The nine examination items included "word recognition," "following simple commands," "visual-spatial perception," "accuracy of the order of a process," "naming fingers," "orientation," "money calculation," "object recognition," and "clock time recognition." The validity of the TDAS has previously been tested and the results showed a significant association between TDAS and ADAS-cog [30]. Scores ranged from 0 (all correct answers) to 101 points (all incorrect answers). A previous study showed that the average TDAS score was approximately 20 points for patients with mild to moderate ADD patients with an average MMSE score of 20 points, approximately 7 points for patients with $\mathrm{MCI}$, and approximately 3 points for patients without cognitive impairment [2]. In contrast, the average TDAS score was shown to be approximately 50 points in individuals with moderate to severe dementia residing in a hospital or nursing home [32].

\section{Dietary and gustatory questionnaire}

We designed a dietary and taste questionnaire. We focused on four items (enjoyment of the meal, deliciousness of the meal, strength of the flavor, and subjective gustatory function) to inquire about palatability of the foods and gustatory function. Each item was self-rated by the participants on a five-point scale (from 0 to 4 points, with 0 indicating the worst and 4 indicating the best).

\section{Measurement of salivary flow}

We measured the saliva secretion volume at rest according to a published protocol [33]. We asked each participant to sit in a quiet room and not to swallow but to continually spit into a receiving tube over a period of 5 min. We then collected the (resting) whole saliva sample. We estimated the volume of saliva by weighing the tubes before and after collection. Different studies have provided different results in terms of the cutoff value for salivary gland hypofunction [33-35]. We used a cutoff value of $<0.2 \mathrm{~mL} / \mathrm{min}$ considering the boundary area.

\section{Blood tests}

Collected blood samples were centrifuged to obtain serum, and the serum samples were immediately stored in polypropylene containers at $-80^{\circ} \mathrm{C}$ until analysis. The levels of total protein (reference: $6.7-8.3 \mathrm{~g} / \mathrm{dL}$ ), albumin (reference: $3.8-5.2 \mathrm{~g} / \mathrm{dL}$ ), and zinc (reference: 64$111 \mu \mathrm{g} / \mathrm{dL}$ ) in the serum were measured by LSI Medience Corporation (Tokyo, Japan). 


\section{Statistical analysis}

We performed all statistical analyses using the SPSS statistical software (version 25; IBM Japan, Tokyo, Japan). We performed Shapiro-Wilk tests to assess data distributions. We assessed differences in the baseline demographics and background characteristics of the participants using one-way analysis of variance (ANOVA), the Kruskal-Wallis test, or the chi-squared test as appropriate. For multiple comparisons, we used the Tukey method or Bonferroni correction. We compared gustatory test scores among the three groups by one-way ANOVA or the Kruskal-Wallis test. The Tukey method or Bonferroni correction was used for multiple comparisons of the degree of change in each group. Furthermore, if the data were normally distributed, the gustatory test scores were compared among the three groups by an analysis of covariance (ANCOVA), with significantly different background characteristics among the three groups as covariates. Consequently, only age was used as a covariate. For multiple comparisons, we used the Bonferroni correction. We applied Pearson's correlation coefficient or Spearman's correlation coefficient to assess correlations between the gustatory test scores and the results of the other tests. All statistical significance tests were two-sided, and we considered alpha-levels of 0.05 as indicative of statistical significance.

\section{Results}

\section{Clinical characteristics}

Table 1 summarizes the characteristics of the participants. Patients in the ADD group were significantly older than those in the MCI and NDC groups (both $p<0.05)$. The TDAS scores were significantly higher in patients with ADD than in those with MCI or NDCs (both $p<0.05$ ). The scores were also significantly higher in MCI compared with those in NDCs $(p<0.05)$. Patients with ADD demonstrated a median TDAS score (interquartile range) of 18 (11-23) points, which suggests mild to moderate ADD. For the other items, we found no significant differences between the three groups.

We found that in each group, participants took a median of 4 to 5 prescription drugs, and participants tended to have polypharmacy. As for body mass index (BMI), the median values were about $22 \mathrm{~kg} / \mathrm{m}^{2}$ in each group, and we found no tendencies for thinness or obesity. We found that the salivary secretion volumes tended to be low in all groups $(<0.2 \mathrm{~mL} / \mathrm{min})$. The total protein, albumin, and zinc in the serum showed a tendency to be low in all groups compared with the reference values. The questionnaire survey showed that most of the participants did not complain about declines in gustatory function and were satisfied with their diet.

\section{Gustatory test}

Figure 1 shows results of gustatory tests (detection and recognition thresholds, and difference between the two). Patients with ADD showed significantly higher detection and recognition thresholds than NDCs according to an ANOVA and the KruskalWallis test (both $p<0.05$ ). Furthermore, we performed an analysis with the age of the subjects, which significantly differed among the three groups, as a covariate. In fact, all data should be performed with an age-adjusted analysis, but due to the complexity of dealing with non-normally distributed variables, we conducted an ANCOVA only for detection threshold, which was the normally distributed variable. The age-adjusted ANCOVA showed a tendency toward significance between patients with ADD and NDCs $(p=0.088)$. On the other hand, the difference between the recognition threshold and the detection threshold was not significantly different among the three groups. Table 2 shows the results for each taste. At the detection threshold of sweet, we found a significant increase in the ADD and MCI groups compared with the NDC group (both $p<0.05$ ). In addition, at the recognition threshold of umami, we found a significant increase in the ADD group compared with the NDC group $(p<0.05)$. Figure 2 shows the cumulative curves of detection and recognition thresholds for each taste in each group. The detection and the recognition threshold curves of each taste tended to shift to the right in patients with MCI and ADD (curves at a higher concentration) compared with those in NDCs. However, the curves of umami were different from those of the other tastes (compared to other tastes, a higher concentration of umami was needed, in all groups). Table 3 shows data on the rate of failure to detect or recognize the highest concentration of a taste solution. In all groups, the percentage of "unrecognizable" responses for umami was the highest compared with other tastes.

\section{Correlations between gustatory test results and other outcomes}

Table 4 shows the results of the correlation analyses between the gustatory test results and age, the number of medications, BMI, the salivary secretion volume, blood test results, and the TDAS score. We found a significant correlation between the detection threshold and BMI $(\mathrm{r}=-0.248, p<0.05)$ and between the recognition threshold and age $(r=0.229, p<0.05)$ and TDAS $(r=0.268, p<0.05)$ in all patients. We also found a significant correlation between age and TDAS $(r=0.324, p=0.002)$. In the analysis results for each diagnostic group, there was only a correlation 
Table 1 Characteristics of patients

\begin{tabular}{|c|c|c|c|c|}
\hline & ADD & $\mathrm{MCl}$ & NDC & $P$ value \\
\hline \multicolumn{5}{|l|}{ Demographics } \\
\hline Number, $\mathrm{n}$ & 29 & 43 & 14 & - \\
\hline Gender (M:F), $\mathrm{n}$ & $9: 20$ & 15:28 & $3: 11$ & 0.641 \\
\hline Age (years), median (IQR) & $83.0(80-87)$ & $79.0(75-84)$ & $74.0(72-78)$ & $0.002^{a}$ \\
\hline Hypertension, \% & 51.7 & 41.9 & 35.7 & 0.557 \\
\hline Dyslipidemia, \% & 24.1 & 30.2 & 28.6 & 0.851 \\
\hline Diabetes mellitus, \% & 10.3 & 7.0 & 14.3 & 0.696 \\
\hline Number of medications, median (IQR) & $5.0(1-6)$ & $4.0(0-7)$ & $5.0(3-7)$ & 0.490 \\
\hline Smoking, \% & 0 & 0 & 7.1 & 0.074 \\
\hline Drinking, \% & 24.1 & 11.6 & 14.3 & 0.361 \\
\hline \multicolumn{5}{|l|}{ Physical examinations, median (IQR) } \\
\hline Height (cm) & $150.1(146-160)$ & $151.0(147-161)$ & $151.5(148-157)$ & 0.809 \\
\hline Weight (kg) & $50.6(45-60)$ & $49.5(45-58)$ & $55.2(50-58)$ & 0.195 \\
\hline Body mass index $\left(\mathrm{kg} / \mathrm{m}^{2}\right)$ & $22.4(20-25)$ & $21.5(20-23)$ & $22.1(21-25)$ & 0.150 \\
\hline Systolic blood pressure (mmHg) & $134.0(126-140)$ & $130.0(128-140)$ & $134.0(126-141)$ & 0.913 \\
\hline Diastolic blood pressure (mmHg) & $74.0(72-80)$ & $74.0(71-80)$ & $77.5(73-86)$ & 0.195 \\
\hline Salivary secretion volume (mL/5 min) & $0.8(0.4-1.4)$ & $0.7(0.4-1.1)$ & $0.9(0.2-1.4)$ & 0.819 \\
\hline \multicolumn{5}{|l|}{ Blood tests, median (IQR) } \\
\hline Total protein (g/dL), ref: $6.7-8.3$ & $7.0(6.6-7.4)$ & $6.9(6.6-7.2)$ & $6.8(6.6-7.3)$ & 0.698 \\
\hline Albumin (g/dL), ref: 3.8-5.2 & $4.0(3.8-4.2)$ & $4.0(3.8-4.2)$ & $4.1(4.0-4.3)$ & 0.426 \\
\hline Zinc $(\mu \mathrm{g} / \mathrm{dL})$, ref: 64-111 & $65.0(57-72)$ & $62.0(55-72)$ & $63.0(59-75)$ & 0.620 \\
\hline \multicolumn{5}{|l|}{ Cognitive test, median (IQR) } \\
\hline TDAS score & $18.0(11-23)$ & $7.0(5-12)$ & $1.0(1-4)$ & $<0.001^{b}$ \\
\hline \multicolumn{5}{|l|}{ Questionnaires, median (IQR) } \\
\hline Subjective gustatory function & $4.0(4-4)$ & $4.0(4-4)$ & $4.0(4-4)$ & 0.891 \\
\hline Enjoyment of the meal & $4.0(4-4)$ & $4.0(3-4)$ & $4.0(4-4)$ & 0.080 \\
\hline Deliciousness of the meal & $4.0(4-4)$ & $4.0(4-4)$ & $4.0(4-4)$ & 0.254 \\
\hline Strength of flavor & $4.0(4-4)$ & $4.0(4-4)$ & $4.0(4-4)$ & 0.443 \\
\hline
\end{tabular}

Gender, hypertension, dyslipidemia, diabetes mellitus, smoking, and drinking ration were analyzed using the chi-squared test. Age, height, diastolic blood pressure, total protein, and zinc were compared by a one-way analysis of variance followed by the Tukey test. Items other than the above were compared by the Kruskal-Wallis test followed by the Bonferroni correction

ADD Alzheimer's disease dementia, MCI mild cognitive impairment, NDC non-demented control, IQR interquartile range, TDAS Touch Panel-type Dementia Assessment Scale, ref reference values

${ }^{a}$ Significant difference between ADD and both $\mathrm{MCI}(p<0.05)$ and NDC $(p<0.05)$

${ }^{\mathrm{b}}$ Significant difference between NDC and MCI $(p<0.05)$, between NDC and AD $(p<0.05)$, and between ADD and MCI $(p<0.05)$

between the detection threshold and zinc in the ADD group $(r=-0.390, p<0.05)$.

\section{Discussion}

In this study, we comprehensively evaluated taste function in patients with ADD or MCI and in NDCs and showed that the taste recognition thresholds were higher in patients with ADD than in the NDCs. This finding is consistent with the results of other studies [8-10]. In terms of the detection threshold, a study investigating the sweet and sour tastes using a whole-mouth gustatory test [19] reported absence of gustatory alterations in patients with ADD. In contrast, the detection threshold was shown to be increased in patients with ADD in a study on the four basic tastes surveyed by the filterpaper disc method $[8,36]$. In this study, we used five basic tastes in 13 different concentrations and found that the overall detection thresholds for various taste solutions showed an increasing trend when we adjusted by age in ADD patients. Therefore, taste detection in the whole oral cavity was considered slightly reduced in ADD patients. In addition, we found no significant differences between the three groups in the comparison of the degree of deviation between the detection and the recognition thresholds, so that the concentration required from detection to recognition did not change, 


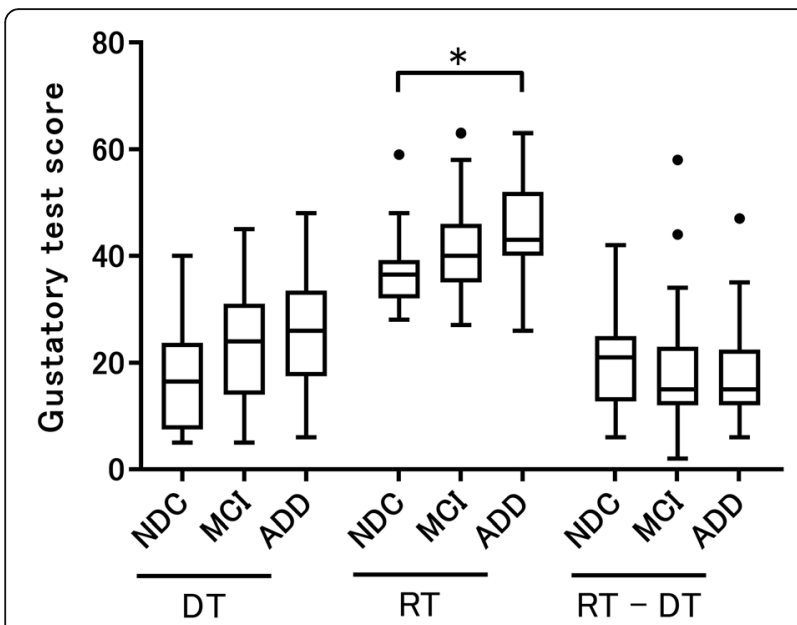

Fig. 1 Results of gustatory tests in patients with Alzheimer's disease dementia (ADD), mild cognitive impairment (MCl), and nondemented controls (NDCs). The recognition threshold (RT) of patients with ADD exhibited a significant increase compared with those in NDCs. However, detection threshold (DT) and RT minus DT (RT - DT) was not significant among three groups. The DT was compared by an analysis of covariance with age as a covariate followed by the Bonferroni correction. The RT and RT - DT were compared by the Kruskal-Wallis test followed by the Bonferroni correction. All data are indicated by box-and-whisker plots following the Tukey method $\left({ }^{*} p<0.05\right)$

and it seemed that the participants responded to tastes appropriately even in the presence of cognitive function decline. On the other hand, we found no significant taste function disorders in patients with MCI, but another study reported a decrease in taste function in these patients [9]. The authors evaluated each region in the oral cavity, but we evaluated the taste perception in the whole oral cavity, so it is possible that taste perceived in the whole oral cavity (as during a meal) is not decreased with MCI.

In our study, many participants could not recognize umami. The recognition threshold of umami was significantly increased in patients with ADD compared with NDCs. Moreover, 69.0 and $44.2 \%$ of patients with ADD and $\mathrm{MCI}$, respectively, could not recognize even the highest concentration of umami solution. This finding suggested the possibility that recognition of umami was the worst in patients with ADD among the three groups. It also suggested that some patients with MCI may have poor recognition of umami. The inability to sense umami is thought to lead to loss of appetite [21], which furthermore leads to a decrease in interest in diet. The amount of overall salivary flow increases with umami taste stimulation by MSG [22], and improving saliva secretion may improve taste perception. In addition, continuous ingestion of MSG has been reported to improve the quality of life and nutritional status of hospitalized elderly patients [37], and to provide benefits to
Table 2 Results of gustatory test for each taste solution

\begin{tabular}{|c|c|c|c|c|c|c|c|}
\hline & \multicolumn{2}{|l|}{ ADD } & \multicolumn{2}{|l|}{$\mathrm{MCl}$} & \multicolumn{2}{|l|}{ NDC } & \multirow{2}{*}{$\begin{array}{l}P \\
\text { value }\end{array}$} \\
\hline & Median & IQR & Median & IQR & Median & IQR & \\
\hline \multicolumn{8}{|l|}{ Sweet } \\
\hline DT & 5 & $4-7$ & 6 & $4-7$ & 3 & $2-4.8$ & $0.026^{\circ}$ \\
\hline RT & 8 & $7-10$ & 7 & $6-9$ & 7.5 & $6-8$ & 0.481 \\
\hline RT - DT & 3 & $0-4$ & 1 & $1-4$ & 3 & $2-6$ & 0.123 \\
\hline \multicolumn{8}{|l|}{ Salty } \\
\hline DT & 4 & $2-5$ & 3 & $2-5$ & 2 & $1-3$ & 0.075 \\
\hline RT & 7 & $6-9$ & 7 & $5.5-8$ & 6 & $6-7$ & 0.234 \\
\hline RT - DT & 4 & $1-6$ & 3 & $2-5$ & 4 & $3-5$ & 0.620 \\
\hline
\end{tabular}

\section{Umami}

$\begin{array}{llllllll}\text { DT } & 9 & 6-11 & 6 & 2-9.5 & 4 & 1-7.8 & 0.038^{\mathrm{b}} \\ \text { RT } & 14 & 12-14 & 13 & 10-14 & 10.5 & 9.3-12 & 0.008^{c} \\ \text { RT - DT } & 4 & 2-7 & 6 & 2-9 & 6 & 3.5-7.8 & 0.623\end{array}$

Sour

$\begin{array}{llllllll}\text { DT } & 4 & 2-6 & 3 & 1.5-5 & 1.5 & 1-4 & 0.064 \\ \text { RT } & 7 & 6-10 & 7 & 6-9.5 & 6 & 5.3-7.8 & 0.273 \\ \text { RT - DT } & 4 & 2-5 & 5 & 2-6 & 3.5 & 2.3-5.8 & 0.495\end{array}$

Bitter

$\begin{array}{llllllll}\text { DT } & 5 & 3-8 & 5 & 3-7 & 3.5 & 1.3-5 & 0.325 \\ \text { RT } & 8 & 6-10 & 7 & 5-8.5 & 6 & 5-7.8 & 0.055 \\ \text { RT - DT } & 4 & 0-5 & 1 & 1-3 & 2 & 1-4 & 0.258\end{array}$

All data were compared via the Kruskal-Wallis test followed by the Bonferroni correction

$A D D$ Alzheimer's disease dementia, $M C l$ mild cognitive impairment, $N D C$ nondemented control, IQR interquartile range, $D T$ detection threshold, $R T$ recognition threshold

${ }^{\text {a }}$ Significant difference between NDC and both MCI $(p<0.05)$ and

$\operatorname{ADD}(p<0.05)$

${ }^{\mathrm{b}}$ No significant difference by multiple comparisons

${ }^{c}$ Significant difference between NDC and ADD $(p<0.05)$

the cognitive function of residents in facilities [32]. Therefore, intervention with MSG for elderly people and patients with dementia is gradually attracting attention. Alternatively, in this study, $21.4 \%$ of the participants in the NDC group were not able to recognize even the highest concentration of umami. A previous study reported that at various concentrations of umami, the correct answer rate regarding its perception in the oral cavity was different between young adults and older individuals [38]. However, another report showed that the results of the evaluation of the umami recognition threshold on each region of the tongue did not differ between young adults and older individuals [39]. There is a disagreement regarding the decline in umami recognition with age; nevertheless, the results of our study suggest that umami recognition worsens with age. However, the detection threshold for umami has also been shown to be increased in individuals with dementias other than ADD [40]. Future studies are needed to determine whether this is a specific change in $\mathrm{AD}$, considering its 

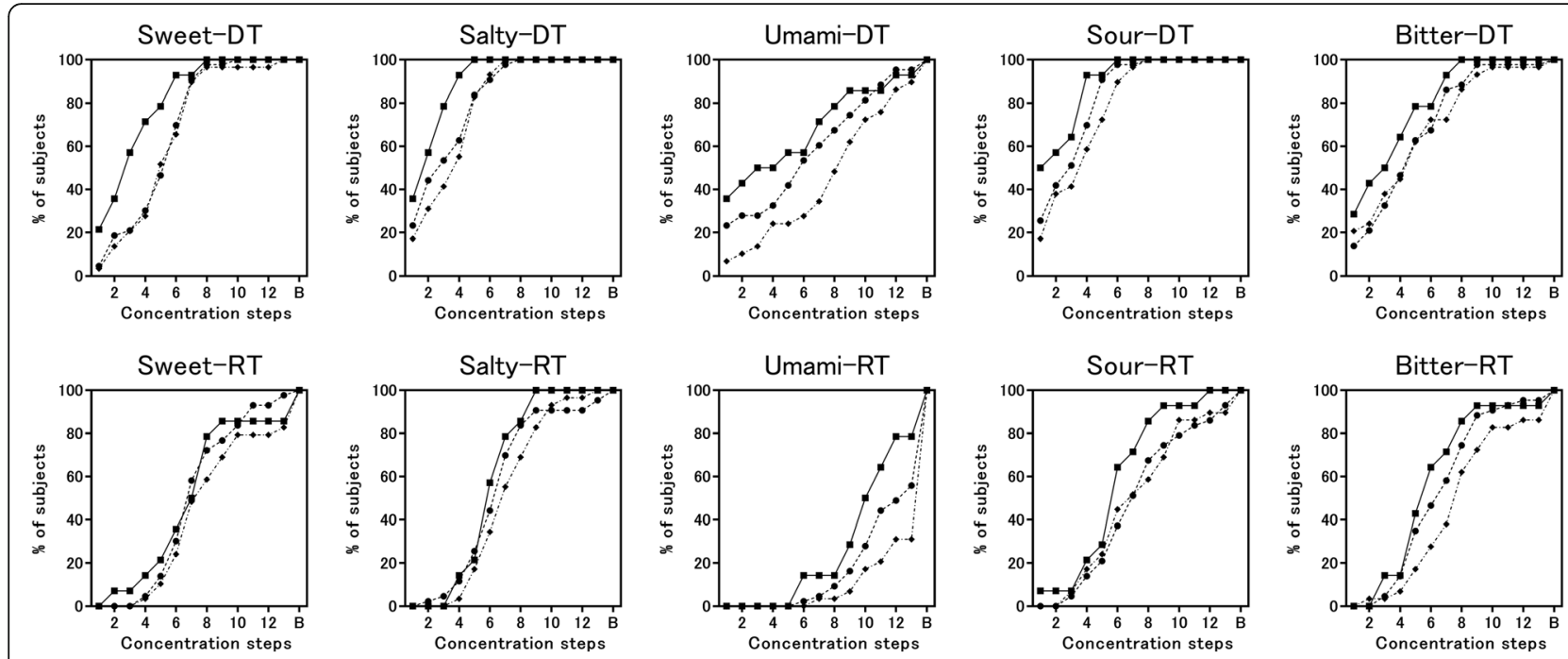

NDC

-- MCI

$\cdots$ ADD

Fig. 2 Cumulative detection threshold (DT) and recognition threshold (RT) curves in patients with Alzheimer's disease dementia (ADD) or mild cognitive impairment $(\mathrm{MCl})$, and that in non-demented controls (NDCs). If the participants could not detect and recognize a taste, even at the highest concentration, those results are indicated as "burst (B)"

association with pathological changes. For tastes other than umami, the non-recognition percentages for sweet and bitter (even at the maximum concentration) were high in ADD. Overall, more participants did not recognize the taste via G-protein coupled receptors than via ion channels. However, whether this difference in transmission format is associated with a failure caused by disease is unknown and needs future study.

In the dietary and gustatory questionnaires, we found few complaints about the decline in the

Table 3 The rate of failure (i.e., burst) to detect or recognize the highest concentration of taste solution

\begin{tabular}{|c|c|c|c|c|c|c|}
\hline & \multicolumn{2}{|c|}{ ADD } & \multicolumn{2}{|c|}{$\mathrm{MCl}$} & \multicolumn{2}{|c|}{ NDC } \\
\hline & $n$ & $\%$ & $n$ & $\%$ & $n$ & $\%$ \\
\hline \multicolumn{7}{|l|}{ Burst of DT } \\
\hline Sweet & 0 & 0 & 0 & 0 & 0 & 0 \\
\hline Salty & 0 & 0 & 0 & 0 & 0 & 0 \\
\hline Umami & 3 & 10.3 & 2 & 4.7 & 1 & 7.1 \\
\hline Sour & 0 & 0 & 0 & 0 & 0 & 0 \\
\hline Bitter & 1 & 3.4 & 1 & 2.3 & 0 & 0 \\
\hline \multicolumn{7}{|l|}{ Burst of RT } \\
\hline Sweet & 5 & 17.2 & 1 & 2.3 & 2 & 14.3 \\
\hline Salty & 0 & 0 & 2 & 4.7 & 0 & 0 \\
\hline Umami & 20 & 69.0 & 19 & 44.2 & 3 & 21.4 \\
\hline Sour & 3 & 10.3 & 3 & 7.0 & 0 & 0 \\
\hline Bitter & 4 & 13.8 & 2 & 4.7 & 1 & 7.1 \\
\hline
\end{tabular}

$A D D$ Alzheimer's disease dementia, $M C l$ mild cognitive impairment, $N D C$ nondemented control, $D T$ detection threshold, $R T$ recognition threshold subjective gustatory function in any group, and many participants answered that they were able to feel the taste of their meals well. Large-scale questionnaires have reported fewer complaints of age-related gustatory dysfunction than of age-related visual, auditory, and olfactory dysfunctions [41], and complete ageusia is rare in subjects with age-related taste function declines $[42,43]$. This may be explained by the fact that taste function is unlikely to be impaired because three different cranial nerves (the facial, glossopharyngeal, and vagus nerves) provide sensory coverage for the tongue and mouth. Furthermore, because the decline in taste function due to dementia seems to be slowly progressive, gradually decreasing function may go unnoticed by individuals. In addition, although the clinical examination tested each taste separately, an actual meal consists of several simultaneous tastes. Therefore, discrepancies between the examination and the subjective gustatory function may exist. No significant differences in the results of the dietary and gustatory questionnaire were considered to exist among the three groups for the above reasons. Furthermore, the absence of any dietary problems in the questionnaire suggests that the ADD group, which experienced decreased taste function, did not experience a decrease in BMI due to weight loss. This study was a cross-sectional study, so it is necessary to conduct a longitudinal study. However, even in the absence of subjective symptoms, the test scores may be low in patients with MCI or ADD; thus, accurate assessments are important. 
Table 4 Correlation between gustatory test results and other test results

\begin{tabular}{|c|c|c|c|c|c|c|c|c|}
\hline & Age & Medications & BMl & Salivation & Total protein & Albumin & Zinc & TDAS \\
\hline \multicolumn{9}{|c|}{ All patients } \\
\hline DT & 0.186 & -0.082 & $-0.248^{*}$ & 0.096 & 0.076 & -0.168 & -0.118 & 0.179 \\
\hline RT & $0.229^{*}$ & 0.078 & -0.067 & 0.177 & 0.132 & -0.112 & -0.065 & $0.268^{*}$ \\
\hline \multicolumn{9}{|l|}{ ADD } \\
\hline DT & 0.061 & -0.217 & -0.041 & 0.146 & 0.004 & -0.322 & $-0.390^{*}$ & -0.008 \\
\hline RT & 0.173 & -0.018 & 0.126 & 0.353 & 0.153 & -0.065 & -0.125 & 0.014 \\
\hline \multicolumn{9}{|l|}{$\mathrm{MCl}$} \\
\hline DT & 0.203 & -0.021 & -0.209 & 0.034 & 0.188 & -0.196 & -0.103 & 0.083 \\
\hline RT & -0.002 & 0.099 & -0.039 & 0.188 & 0.243 & -0.150 & -0.084 & 0.087 \\
\hline \multicolumn{9}{|l|}{ NDC } \\
\hline DT & -0.141 & 0.332 & -0.463 & 0.139 & -0.204 & 0.201 & 0.194 & 0.096 \\
\hline RT & 0.119 & 0.449 & -0.128 & -0.281 & -0.412 & 0.090 & -0.085 & 0.133 \\
\hline
\end{tabular}

In all patients, correlations between DT and age and total protein were analyzed using Pearson's correlation coefficient, and other correlation analyses were conducted using Spearman's correlation coefficient. In patients with ADD, correlations between DT or RT, and age, BMI, total protein, zinc, and TDAS score were analyzed using Pearson's correlation coefficient, and other correlation analyses were conducted using Spearman's correlation coefficient. In patients with MCI, correlations between DT or RT and age, total protein, albumin, and zinc were analyzed using Pearson's correlation coefficient, and other correlation analyses were conducted using Spearman's correlation coefficient. In NDCs, correlations between DT and age, medications, salivation, total protein, albumin, and zinc were analyzed using Pearson's correlation coefficient, and other correlation analyses were conducted using Spearman's correlation coefficient $A D D$ Alzheimer's disease dementia, $M C l$ mild cognitive impairment, $N D C$ non-demented control, $D T$ detection threshold, $R T$ recognition threshold, $B M I$ body mass index, TDAS Touch Panel-type Dementia Assessment Scale

Data are presented correlation coefficient $(r) .{ }^{*} p<0.05$

We found a correlation between taste detection and BMI, suggesting that slender individuals may have difficulty perceiving tastes in this study. An association between taste disorders and malnutrition has been reported in the elderly [44], and nutritional bias is thought to lead to zinc deficiency [45] and eventually affect taste. However, there was a large difference in the correlation coefficient between taste detection and BMI among the three groups; MCI and NDCs, had particularly large correlation coefficients. Another factor may be involved in patients with ADD. It is necessary to increase the number of cases to make further conclusions. On the other hand, taste recognition is thought to be associated with age and cognitive function, and cognitive function was not associated with the detection threshold but rather with the recognition threshold. Similar to a previous study [8], the cause of the decline in taste function in dementia is not thought to be due to impaired transmission from peripheral receptors but to a decrease in the taste perception cognitive ability that accompanies brain atrophy and neurodegeneration. However, patients with ADD showed a significant increase in the detection threshold compared with NDCs, so we think that taste detection may be affected by brain disorders. In fact, the insular cortex/frontal operculum has been shown to be activated by water stimulation [46], and some areas of the brain may be involved in taste detection. In addition, a study examining the taste function in patients with semantic dementia, which damages the insular cortex, showed that there is an increase in taste detection thresholds [36]. Moreover, the density of insular neurofibrillary tangles has been significantly positively correlated with the duration of clinical dementia [47].

Many of the participants in this study had decreased salivary secretion as well as low serum zinc levels. The average number of prescription medications in geriatric outpatients at five university hospitals has been estimated to be between 3 and 5 [48]. In this study, the results were roughly similar, and the median of the number of prescription medications was 4-5 in each group of participants. Many individuals were on multiple medications. The decrease in taste function in the elderly is thought to be the result of many factors, including decreased salivary secretion, decreased serum zinc levels, effects of comorbidities, and multidrug use [22, 24, 49]. In this study, we found no significant differences in terms of those variables among the three groups. Therefore, we think the decline in taste function due to $\mathrm{AD}$ was caused by factors inherent to the disease and not to age.

Finally, we are aware of the limitations of our study. The first is related to our scoring. Because many participants did not recognize even the maximum concentration of umami, the score reached its peak prematurely, and we cannot rule out that a higher peak may have shown differences between the groups. However, the clinical significance of using a much higher concentration of taste solutions must be carefully considered. The second limitation of our study concerns the age of the participants. Compared with NDCs and patients with 
MCI, patients with ADD were significantly older. The detection threshold did not significantly differ when we adjusted by age. Gustatory function has been shown to decline with age [38, 42, 50-52], but progression of gustatory hypofunction in elderly (especially after 75 years of age) still remains unclear. We found a correlation between age and taste recognition threshold, but age was also correlated with the cognitive function, and older patients had more severe cognitive disorders. Because older patients have cognitive disorders, it was thought that the gustatory function decreased because of brain disorders that caused cognitive impairment. In addition, there was no significant correlation between age and gustatory test scores in each group, which suggests that aging may be an intermediate factor that promotes factors affecting taste function, such as brain disorders, decreased salivary secretion, decreased serum zinc levels, effects of comorbidities, and multidrug use. All results were to be analyzed by ANCOVA with age as a covariate, but ANCOVA was rarely able to be performed because many results were not normally distributed. The third limitation is that worse gustatory test scores might also be a consequence of cognitive disorders rather than gustatory dysfunction. The presence of multimodal semantic impairment in ADD is well known. Semantic deficits have been reported to potentially reduce olfactory test scores in patients with ADD [3]. In addition, the taste-to-picture matching task score for patients with semantic dementia has been shown to be significantly lower than that of patients with ADD or control patients [36]. Therefore, it is possible that semantic impairment reduced the gustatory test score. However, it was reported that a taste test, in which choices by letter were selected (similar to the method used in this study), showed no significant difference in the scores between patients with semantic dementia and patients with ADD [36], and it is possible that the impact of semantic impairment may be smaller in this study. We believe that, in the future, it will be necessary to verify whether deteriorations in the gustatory test score are due to brain degeneration or cognitive decline. Finally, the number of participants was relatively low. The NDCs were targeted outpatients in the hospital diagnosed as not having dementia, so recruitment was difficult. However, we selected participants according to the exact doctor's diagnostic criteria, and we believe that the reliability of the results is high.

\section{Conclusions}

In this study, the gustatory function of patients with $\mathrm{ADD}$ and $\mathrm{MCI}$, and that of NDCs for five basic tastes was evaluated by the whole-mouth gustatory test. We confirmed taste function declines in patients with ADD, but not in MCI, suggesting the dysfunction of taste is not seen in the early stage of the disease. Salivary secretion, serum zinc levels, and multidrug intake were not correlated with gustatory decline, suggesting that these factors are unlikely to explain our results. However, many patients with ADD or MCI were not able to recognize umami, which decreased their interest in meals, and this variable should be longitudinally studied. Taste function is an indispensable sensory function for adjustment of intake of nutrients necessary for the body and to successfully reject foreign substances. We believe that detecting and interfering with impaired gustatory function at an early stage of recognition impairment may improve dietary interest and quality of life.

\section{Abbreviations \\ ADD: Alzheimer's disease dementia; MCl: Mild cognitive impairment; NDCs: Non-demented controls; ADAS-Cog: Alzheimer's Disease Assessment Scale-Cognitive Subscale; CT: Computed tomography; TDAS: Touch Panel- type Dementia Assessment Scale; BMI: Body mass index; ANOVA: Analysis of variance; ANCOVA: Analysis of covariance}

\section{Acknowledgments}

We thank the patients who participated in our study and their families. We also thank the staff at Shinsei Hospital (Kurayoshi, Japan) who cooperated with this study.

\section{Authors' contributions}

MK, HM, HC, and KU designed the study. MK, Jl, DS, FK, and YK implemented the tests. MK analyzed the data and MK, SY, and KU interpreted the data. MK wrote the first draft of the manuscript and HC, SY, and KU reviewed the manuscript. All authors read and approved the final manuscript.

\section{Funding}

This study was funded by Ajinomoto. The funding body participated in the design of the study, interpretation of data and preparation of the manuscript.

\section{Availability of data and materials}

The datasets used and/or analysed during the current study are available from the corresponding author on reasonable request. However, the available of datasets is only possible if all authors and the ethics committee approve.

Ethics approval and consent to participate

The ethics committee of Tottori University (Yonago, Japan) and Ajinomoto (Tokyo, Japan) approved the design of this study (approval number:

1701B073), conducted by following the tenets of the Declaration of Helsinki. We explained the research protocol to the patients and guardians, who provided written informed consents for participation.

\section{Consent for publication}

Not applicable.

\section{Competing interests}

MK, JI, DS, FK, and YK have no conflicts of interest to declare. KU owns a patent on the Touch Panel-type Dementia Assessment Scale and receives royalties from Nihon Kohden Corporation. HM, HC, and SY are employees of Ajinomoto.

\section{Author details}

${ }^{1}$ Department of Biological Regulation, School of Health Science, Faculty of Medicine, Tottori University, 86 Nishi-cho, Yonago 683-8503, Japan. ${ }^{2}$ Research Institute for Bioscience \& Fine Chemicals, Ajinomoto Co., Inc., 1-1, Suzuki-Cho, Kawasaki-Ku, Kawasaki-Shi 210-8681, Japan. 


\section{Received: 14 November 2019 Accepted: 18 March 2020}

Published online: 26 March 2020

\section{References}

1. Kotecha AM, Corrêa ADC, Fisher KM, Rushworth JV. Olfactory Dysfunction as a Global Biomarker for Sniffing out Alzheimer's Disease: A Meta-Analysis. Biosensors (Basel). 2018; 8: pii: E41.

2. Kouzuki M, Suzuki T, Nagano M, Nakamura S, Katsumata Y, Takamura A, et al. Comparison of olfactory and gustatory disorders in Alzheimer's disease. Neurol Sci. 2018;39:321-8.

3. Djordjevic J, Jones-Gotman M, De Sousa K, Chertkow H. Olfaction in patients with mild cognitive impairment and Alzheimer's disease. Neurobiol Aging. 2008:29:693-706.

4. Kjelvik G, Saltvedt I, White LR, Stenumgård P, Sletvold O, Engedal K, et al. The brain structural and cognitive basis of odor identification deficits in mild cognitive impairment and Alzheimer's disease. BMC Neurol. 2014;14:168.

5. Loughrey DG, Kelly ME, Kelley GA, Brennan S, Lawlor BA. Association of ageRelated Hearing Loss with Cognitive Function, cognitive impairment, and dementia: a systematic review and meta-analysis. JAMA Otolaryngol Head Neck Surg. 2018;144:115-26.

6. Golub JS, Luchsinger JA, Manly JJ, Stern Y, Mayeux R, Schupf N. Observed hearing loss and incident dementia in a multiethnic cohort. J Am Geriatr Soc. 2017;65:1691-7.

7. Gallacher J, llubaera V, Ben-Shlomo Y, Bayer A, Fish M, Babisch W, et al. Auditory threshold, phonologic demand, and incident dementia. Neurology. 2012:79:1583-90.

8. Ogawa T, Irikawa N, Yanagisawa D, Shiino A, Tooyama I, Shimizu T. Taste detection and recognition thresholds in Japanese patients with Alzheimertype dementia. Auris Nasus Larynx. 2017:44:168-73.

9. Steinbach S, Hundt W, Vaitl A, Heinrich P, Förster S, Bürger $K$, et al. Taste in mild cognitive impairment and Alzheimer's disease. J Neurol. 2010;257:238-46.

10. Sakai M, Ikeda M, Kazui H, Shigenobu K, Nishikawa T. Decline of gustatory sensitivity with the progression of Alzheimer's disease. Int Psychogeriatr. 2016;28:511-7

11. Kai K, Hashimoto M, Amano K, Tanaka H, Fukuhara R, Ikeda M. Relationship between eating disturbance and dementia severity in patients with Alzheimer's disease. PLoS One. 2015;10:e0133666.

12. Tomita $\mathrm{H}$, Ikeda M, Okuda Y. Basis and practice of clinical taste examinations. Auris Nasus Larynx. 1986;13(Suppl 1):S1-15.

13. Mueller C, Kallert S, Renner B, Stiassny K, Temmel AF, Hummel T, et al. Quantitative assessment of gustatory function in a clinical context using impregnated "taste strips". Rhinology. 2003;41:2-6.

14. Landis BN, Welge-Luessen A, Brämerson A, Bende M, Mueller CA, Nordin S, Hummel T. "taste strips" - a rapid, lateralized, gustatory bedside identification test based on impregnated filter papers. J Neurol. 2009;256: 242-8.

15. Murphy C, Quiñonez C, Nordin S. Reliability and validity of electrogustometry and its application to young and elderly persons. Chem Senses. 1995;20:499-503.

16. Yamauchi Y, Endo S, Sakai F, Yoshimura I. A new whole-mouth gustatory test procedure. 1. Thresholds and principal components analysis in healthy men and women. Acta Otolaryngol Suppl. 2002;546:39-48.

17. Yamauchi $Y$, Endo S, Yoshimura I. A new whole-mouth gustatory test procedure. II. Effects of aging, gender and smoking. Acta Otolaryngol Suppl. 2002:546:49-59.

18. Suto T, Meguro K, Nakatsuka M, Kato Y, Tezuka K, Yamaguchi S, et al. Disorders of "taste cognition" are associated with insular involvement in patients with Alzheimer's disease and vascular dementia: "memory of food is impaired in dementia and responsible for poor diet". Int Psychogeriatr. 2014;26:1127-38

19. Koss E, Weiffenbach JM, Haxby JV, Friedland RP. Olfactory detection and identification performance are dissociated in early Alzheimer's disease. Neurology. 1988;38:1228-32.

20. Lang CJ, Leuschner T, Ulrich K, Stössel C, Heckmann JG, Hummel T. Taste in dementing diseases and parkinsonism. J Neurol Sci. 2006;248:177-84.

21. Satoh-Kuriwada S, Kawai M, Shoji N, Sekine $Y$, Uneyama H, Sasano T. Assessment of Umami Taste Sensitivity. J Nutr Food Sci. 2012. https://doi. org/10.4172/2155-9600.S10-003.

22. Sasano T, Satoh-Kuriwada S, Shoji N, likubo M, Kawai M, Uneyama H, et al. Important role of umami taste sensitivity in oral and overall health. Curr Pharm Des. 2014;20:2750-4.
23. Aps JK, Martens LC. Review: the physiology of saliva and transfer of drugs into saliva. Forensic Sci Int. 2005;150:119-31.

24. Ikeda M, Ikui A, Komiyama A, Kobayashi D, Tanaka M. Causative factors of taste disorders in the elderly, and therapeutic effects of zinc. J Laryngol Otol. 2008;122:155-60

25. Ackerman $\mathrm{BH}$, Kasbekar N. Disturbances of taste and smell induced by drugs. Pharmacotherapy. 1997;17:482-96.

26. Naik BS, Shetty N, Maben EV. Drug-induced taste disorders. Eur J Intern Med. 2010;21:240-3.

27. American Psychiatric Association. Diagnostic and Statistical Manual of Mental Disorders. 5th ed. Arlington: American Psychiatric Association; 2013.

28. Petersen RC. Mild cognitive impairment as a diagnostic entity. J Intern Med. 2004:256:183-94.

29. Tsutsumi R, Goda M, Fujimoto C, Kanno K, Nobe M, Kitamura Y, et al. Effects of chemotherapy on gene expression of lingual taste receptors in patients with head and neck cancer. Laryngoscope. 2016;126:E103-9.

30. Inoue $\mathrm{M}$, Jimbo D, Taniquchi M, Urakami K. Touch panel-type dementia assessment scale: a new computer-based rating scale for Alzheimer's disease. Psychogeriatrics. 2011;11:28-33.

31. Rosen WG, Mohs RC, Davis KL. A new rating scale for Alzheimer's disease. Am J Psychiatry. 1984;141:1356-64

32. Kouzuki M, Taniguchi M, Suzuki T, Nagano M, Nakamura S, Katsumata $Y$, et al. Effect of monosodium L-glutamate (umami substance) on cognitive function in people with dementia. Eur J Clin Nutr. 2019;73:266-75.

33. Longman LP, McCracken CF, Higham SM, Field EA. The clinical assessment of oral dryness is a significant predictor of salivary gland hypofunction. Oral Dis. 2000;6:366-70.

34. Navazesh M, Christensen C, Brightman V. Clinical criteria for the diagnosis of salivary gland hypofunction. J Dent Res. 1992;71:1363-9.

35. Sreebny LM, Valdini A. Xerostomia. Part I: relationship to other oral symptoms and salivary gland hypofunction. Oral Surg Oral Med Oral Pathol. 1988:66:451-8.

36. Sakai M, Kazui H, Shigenobu K, Komori K, Ikeda M, Nishikawa T. Gustatory dysfunction as an early symptom of semantic dementia. Dement Geriatr Cogn Dis Extra. 2017;7:395-405.

37. Tomoe M, Inoue Y, Sanbe A, Toyama K, Yamamoto S, Komatsu T. Clinical trial of glutamate for the improvement of nutrition and health in the elderly. Ann N Y Acad Sci. 2009;1170:82-6.

38. Mojet J, Christ-Hazelhof E, Heidema J. Taste perception with age: generic or specific losses in threshold sensitivity to the five basic tastes? Chem Senses. 2001;26:845-60

39. Satoh-Kuriwada S, Kawai M, likubo M, Sekine-Hayakawa Y, Shoji N, Uneyama $\mathrm{H}$, et al. Development of an umami taste sensitivity test and its clinical use. PLoS One. 2014;9:e95177.

40. Schiffman SS, Clark CM, Warwick ZS. Gustatory and olfactory dysfunction in dementia: not specific to Alzheimer's disease. Neurobiol Aging. 1990;11: 597-600.

41. Michikawa T, Nishiwaki Y, Takebayashi T. Are you conscious of any agerelated taste impairment? Prevalence of and factors associated with taste impairment in Japan. J Am Geriatr Soc. 2011;59:951-3.

42. Welge-Lüssen A, Dörig P, Wolfensberger M, Krone F, Hummel T. A study about the frequency of taste disorders. J Neurol. 2011;258:386-92.

43. Mann NM. Management of smell and taste problems. Cleve Clin J Med. 2002;69:329-36.

44. Vanderwee K, Clays E, Bocquaert I, Gobert M, Folens B, Defloor T. Malnutrition and associated factors in elderly hospital patients: a Belgian cross-sectional, multi-Centre study. Clin Nutr. 2010;29:469-76.

45. Wapnir RA. Zinc deficiency, malnutrition and the gastrointestinal tract. J Nutr. 2000;130:1388S-92S

46. Kobayashi M, Takeda M, Hattori N, Fukunaga M, Sasabe T, Inoue N, et al. Functional imaging of gustatory perception and imagery: "top-down" processing of gustatory signals. Neuroimage. 2004;23:1271-82.

47. Bonthius DJ, Solodkin A, Van Hoesen GW. Pathology of the insular cortex in Alzheimer disease depends on cortical architecture. J Neuropathol Exp Neurol. 2005;64:910-22.

48. Suzuki Y, Akishita M, Arai H, Teramoto S, Morimoto S, Toba K. Multiple consultations and polypharmacy of patients attending geriatric outpatient units of university hospitals. Geriatr Gerontol Int. 2006;6:244-7.

49. Kinugasa Y, Nakayama N, Sugihara S, Mizuta E, Nakamura K, Kamitani H, et al. Polypharmacy and taste disorders in heart failure patients. Eur J Prev Cardiol. 2019. https://doi.org/10.1177/2047487319856717. 
50. Yoshinaka M, Ikebe K, Uota M, Ogawa T, Okada T, Inomata C, et al. Age and sex differences in the taste sensitivity of young adult, young-old and oldold Japanese. Geriatr Gerontol Int. 2016;16:1281-8.

51. Fukunaga A, Uematsu H, Sugimoto K. Influences of aging on taste perception and oral somatic sensation. J Gerontol A Biol Sci Med Sci. 2005 60:109-13.

52. Methven L, Allen VJ, Withers CA, Gosney MA. Ageing and taste. Proc Nutr Soc. 2012;71:556-65.

\section{Publisher's Note}

Springer Nature remains neutral with regard to jurisdictional claims in published maps and institutional affiliations.

- fast, convenient online submission

- thorough peer review by experienced researchers in your field

- rapid publication on acceptance

- support for research data, including large and complex data types

- gold Open Access which fosters wider collaboration and increased citations

- maximum visibility for your research: over $100 \mathrm{M}$ website views per year

At BMC, research is always in progress. 